\section{SENSING RAIL SYSTEM WITH PIEZOELECTRIC ELEMENTS}

ZDENEK MACHU ${ }^{1}$, MARTINA KRATOCHVILOVA ${ }^{2}$, FILIP KSICA ${ }^{1}$, JAN PODROUZEK ${ }^{2}$, ZDENEK HADAS ${ }^{1}$

${ }^{1}$ Faculty of Mechanical Engineering, Brno University of Technology, Brno, Czech Republic

${ }^{2}$ Faculty of Civil Engineering, Brno University of Technology, Brno, Czech Republic

DOI : 10.17973/MMSJ.2021_03_2020066

e-mail : kratochvilova.m@fce.vutbr.cz

This paper deals with piezoelectric strain measurement for rail monitoring applications. Piezoelectric materials under mechanical load generate electric charge and thus provide active voltage response. Mechanical parts with piezoelectric sensing layers could provide electrical signal which is proportional with mechanical loads and vibration. Presented piezoelectric system in the form of a macro fiber composite (MFC) or polymer polyvinylidene fluoride (PVDF) could be used for monitoring applications. A lab application shows the potential of these sensors and the piezoelectric response is compared with the strain gauge measurement. A real application on a regional track is proposed and the piezoelectric patch is integrated on the rail. Analysis of the obtained voltage signal is compared with the traditional acceleration analysis. Proposed solution provides an affordable sensing system which could monitor and detect system wear or maximal deformation under operation.

KEYWORDS

Piezoelectric, PVDF, MFC, Strain Sensor, Rail, Deformation, Monitoring

\section{INTRODUCTION}

Recent development of modern Industry 4.0 applications expect higher availability and affordability of sensors, data acquisition systems and big data technologies [Lee 2015]. Also modern train transportation requires advanced sensing systems for trackside monitoring due to safety reasons and automatization of track condition monitoring. A regular visual inspection of the railways condition does not provide a workable solution for modern high traffic density railways. This paper suggests an affordable sensing solution for track monitoring which is based on piezoelectric materials. Piezoelectric patches or layers could be integrated on rails and it means that it could be used with the current infrastructure. The adaptation of piezoelectric layers could be used for monitoring in many engineering applications under dynamic loads.

Piezoelectric elements and materials are commonly used in smart structural systems as active sensors of mechanical operation. Mechanical strain could be measured in terms of the charge generated by the piezoelectric element as a result of the direct piezoelectric effect [Sirohi 2000]. Piezoelectric materials under dynamic mechanical load provide active voltage response and it could be used as sensing layer in cyber-physical systems. The presented approach for monitoring of rail deformation could be adopted for many engineering applications which are in accordance with Industry 4.0 methodology.

\section{STATE OF ART IN RAIL MONITORING}

Rail transport as a part of Trans-European Transport Networks (TEN-T) [Pereira 2019] is an important element of infrastructure. Its advantages lie mainly in safety, easy organizability, long- distance speed and, last but not least, it is very advantageous for the transport of heavy goods. However, the conditions under which railways operate have changed greatly in recent decades. The traffic of the lines is increasing, and the trains are transporting more and more loads. Therefore, the dynamic effects caused by them on the tracks are also higher. Climate changes also have a further effect on reducing the life of railways [Koetse 2009, Oslakovic 2012]. Previous research has shown that 5-10\% of failures are caused by weather (e.g. temperature and extreme events such as thunderstorms) [Oslakovic 2012]. Due to the long period of regular visual inspections of the condition of the railways (e.g. every year), it takes a long time to track the changes and thus the possibility of failure. Eventually a failure may occur between inspections. The poor condition of railways not only increases costs and reduces the comfort of their use but can also damage property and health. With the current idea of Structural Health Monitoring (SHM), there is an effort to replace visual inspections with sensors that continuously monitor some characteristics and based on their values (changes) it is possible to evaluate the current state and the need for possible maintenance. SHM is a very current topic in many other engineering fields such as mechanical engineering [Salameh 2018], civil infrastructure [Chen 2018], aviation industry [Qing 2019] and others.

There are many types of sensors used in SHM [Ngamkhanong 2018]. Among others, it is possible to divide them according to the way of their placement into movable and fixed. Movable or are those that are placed on the moving object (train) [Li 2017]. In contrast, fixed ones are statically attached to the rails. The sensors can be further divided into wired and wireless. For fixed monitoring can be used wired sensors. However these are more time consuming to install and can be even more expensive [Akyildiz 2002]. For wireless sensors, data obtained on individual sensor nodes are collected using Wi-Fi, Bluetooth or others on the base station from where they are sent via long-range communication and LTE, GPRS, EDGE to the server [Hodge 2015]. However, the disadvantage is that each sensor must have its own battery and its endurance is strongly influenced by the manner and frequency of data transmission. Especially on busy railway tracks, frequent battery replacement is complicated, and therefore the development of sensor node power systems is a very actual issue. For example, with the help of newly developed harvesting technologies it is possible to locally generate electrical energy from the environment to create maintenancefree wireless system networks [Tang 2018].

The frequently measured characteristic in the railway application of SHM is the acceleration derived from vibration of the track excited by the passage of the train. Accelerometers can be located at various points on the rail (or even below it) and measure acceleration in both the horizontal and vertical planes [Lucero 2019, Zhang 2015]. These devices can use various technologies, such as piezoelectricity, capacitance, laser based, magnetic induction, optical, resonance and others [I. Lee 2005]. Currently, the most commonly used accelerometers with SHM of railways are Micro electromechanical systems (MEMS) [Andrejašic 2008, Milne 2016]. Their advantage is small size and ability to work in demanding conditions [Krebs 2015].

Data obtained from accelerometers can be converted to force and then wheel defects can be determined [Barke 2006]. Another common procedure is to convert acceleration to displacements [Milne 2018]. This can be done in the time or frequency domain [Brandt 2014]. In the first case, the measured time series are double integrated. However resulting displacement signal is affected by drift of the baseline caused by noise, random errors and unknown initial values. For these reasons, many baseline correction methods have been 
developed [Thong 2004, Trifunac 2001]. The second method is data processing in the frequency domain. However the Fourier transform is used and so the large truncation error is introduced [Zhu 2015]. Because displacements are an important characteristic of changes in the condition of a structure, their calculation is a major challenge in many industries [Abir 2016, Park 2005, Yang 2006].

To overcome the errors caused by signal processing of accelerometer data, in this work we shall introduce a piezoelectric sensor which can be effectively used to measure strain rate on the rail upon train passing and further use the measured strain rate to estimate the rail deflection at measured location.

\section{ANALYSIS OF RAIL ACCELERATION MEASUREMENT}

An estimation of a rail deformation under moving load of a passing train is basic analysis which can be done from acceleration measurements. However due to noise and double numerical integration it could be a very difficult task for estimation of rail displacement under train. The acceleration measurement was done on a regional track, see Fig. 1, where a single car regional train with two axles passed with speed around $27 \mathrm{~km} / \mathrm{h}$. An accelerometer with $50 \mathrm{~g}$ range was used and placed on the rail, positioned in the middle between sleepers.

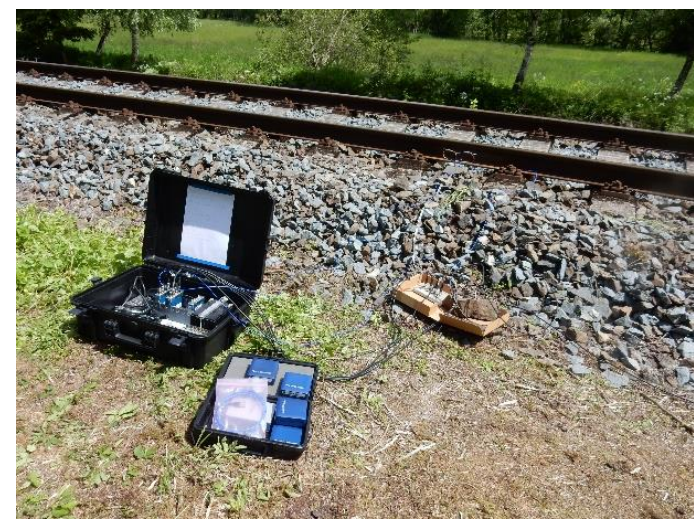

Figure 1. Acceleration measurement on a regional track.

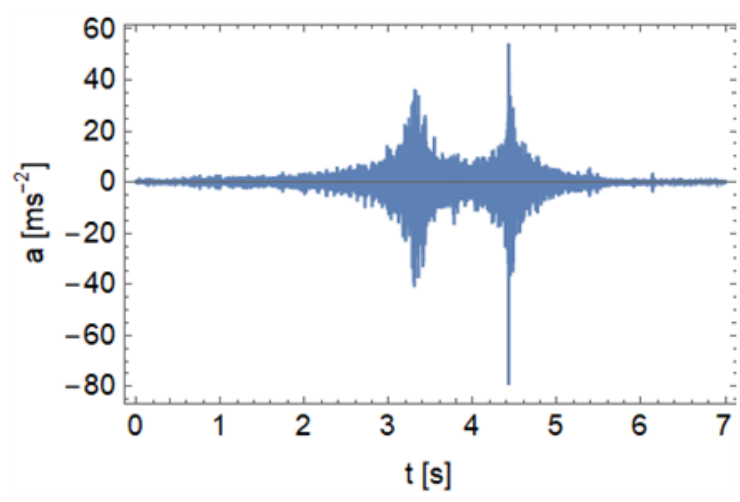

Figure 2. Acceleration measured signal from experiment.

Fig. 2 shows the time series of the acceleration over time obtained from the experiment. For the needs of SHM, it is appropriate to convert the acceleration to displacement, because it is an easier-to-interpret characteristic. From its values it is possible to determine, for example, changes in the stiffness of the support. As mentioned above, this can be done in either the time or frequency domain. However, the measured acceleration data already contains errors from the analog-to- digital conversion and from the design limitations of the measuring devices and some noise.

\subsection{Time domain analysis}

Fig. 3 and Fig. 4 show the results of the first and second integration of the time series, i.e. speed and displacements. It is obvious that already by speed time series the baseline is already drifted and rotated, and thus the results of the second integration give completely unrealistic results. For railway applications, according to the authors' knowledge, there is currently no reliable method for correcting this drift and rotation, because the initial conditions are seldom known.

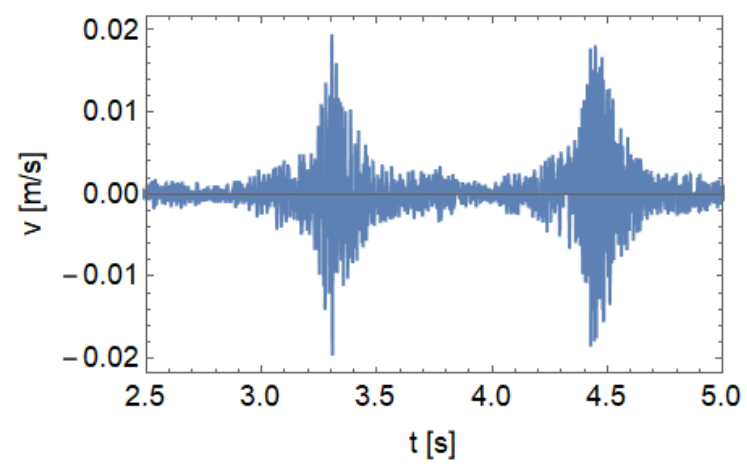

Figure 3. Result of the first integration in time domain.

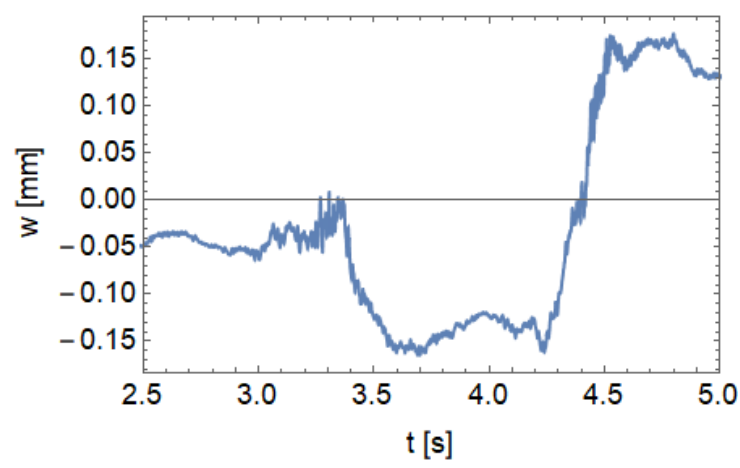

Figure 4. Result of the second integration in time domain.

\subsection{Frequency domain analysis}

The data is transferred to the frequency domain using the Fourier Transform. The results are shown in Fig. 5. As the double integration is then performed also in frequency domain, the integrals are transformed to an algebraic operation.

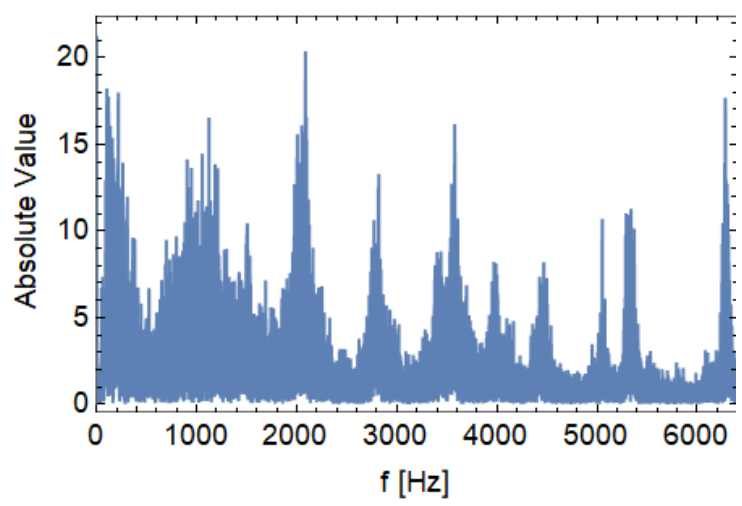

Figure 5. Acceleration data after Fourier Transform. 
Results in time domain are then obtained using Inverse-Fourier Transform and it is shown in Fig. 6.

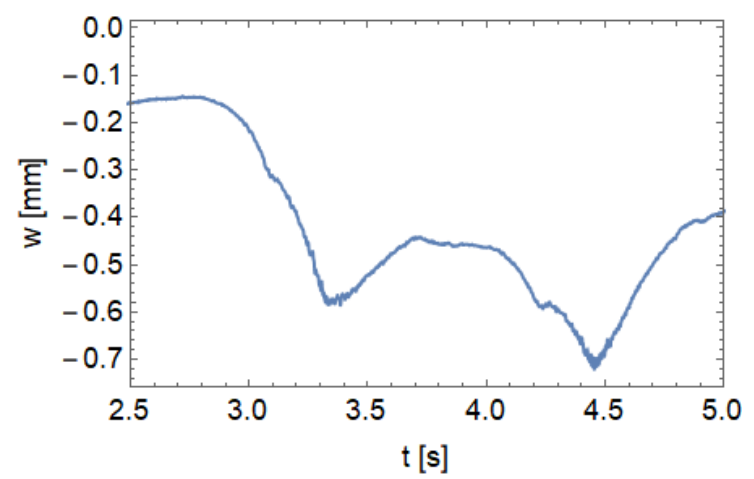

Figure 6. Result of the second integration in frequency domain.

It is known from the history of the experiment that the rail has returned to its original position, so the displacements at the end of the measured period should be zero. Thus, even the results obtained from the integration in the frequency plane do not correspond to reality. Unfortunately, it has not yet been possible to reliably determine which frequencies are significant and which are only noise and can therefore be filtered.

\section{UTILIZING PIEZOELECTRICITY FOR STRAIN MEASUREMENT}

Piezoelectricity is a property of certain materials to generate electric charge when deformed or, in reverse, to deform when an external electric field is applied. This happens due to a particular crystallographic symmetry within the crystal lattice [Kholkin 2008]. In technical practice, ferroelectric piezo-ceramics [Cochran 2012], piezo-polymers [Sappati 2018] or piezo composites [Płaczek 2019] are used in various applications due to their superior piezoelectric and particular mechanical properties. All of these commonly used materials have a one common property: axis of polarization. Polarization allows for creating of a unidirectional internal electric field within the piezoelectric material along the polarization axis when a normal deformation is applied. If metal conducting layers (electrodes) are attached to surfaces perpendicular to polarization axis, one can collect the generated electric charge. When the applied deformation varies in time, then the piezoelectric material with electrodes acts as a source of electric current which can be used with an advantage to design an autonomous sensing device. It could be used in open circuit measurement or with a specified resistive load $R_{l}$ which is sensitive to time-related changes of normal strain in a specified direction. On the other hand, the ambient temperatures encountered upon the measurement are assumed not to alter the piezoelectric parameters. Also, the very short train sensing time means that pyroelectric effect does not occur, and thus it cannot affect the voltage response.

\subsection{Theory: Strain of piezoelectric element}

Here, we shall derive the relationship between the voltage generated by a piezoelectric element and the strain rate detected by a piezoelectric element. Under the assumption that piezoelectric behavior of used materials is linear, following relations between electrical and mechanical quantities apply [Meitzler 1988]:

$$
\begin{aligned}
\left\{\begin{array}{l}
\varepsilon_{x} \\
\varepsilon_{y} \\
\varepsilon_{z} \\
\gamma_{y z} \\
\gamma_{x z} \\
\gamma_{x y}
\end{array}\right\}=\left[\begin{array}{cccccc}
s_{11}^{E} & s_{12}^{E} & s_{13}^{E} & 0 & 0 & 0 \\
s_{21}^{E} & s_{22}^{E} & s_{23}^{E} & 0 & 0 & 0 \\
s_{31}^{E} & s_{32}^{E} & s_{33}^{E} & 0 & 0 & 0 \\
0 & 0 & 0 & s_{44}^{E} & 0 & 0 \\
0 & 0 & 0 & 0 & s_{55}^{E} & 0 \\
0 & 0 & 0 & 0 & 0 & s_{66}^{E}
\end{array}\right]\left\{\begin{array}{c}
\sigma_{x} \\
\sigma_{y} \\
\sigma_{z} \\
\tau_{y z} \\
\tau_{x z} \\
\tau_{x y}
\end{array}\right\} \\
+\left[\begin{array}{ccc}
0 & 0 & d_{31} \\
0 & 0 & d_{31} \\
0 & 0 & d_{33} \\
0 & d_{15} & 0 \\
d_{15} & 0 & 0 \\
0 & 0 & 0
\end{array}\right]\left\{\begin{array}{l}
E_{x} \\
E_{y} \\
E_{z}
\end{array}\right\}
\end{aligned}
$$

$$
\begin{aligned}
\left\{\begin{array}{l}
D_{x} \\
D_{y} \\
D_{z}
\end{array}\right\}=\left[\begin{array}{cccccc}
0 & 0 & 0 & 0 & d_{15} & 0 \\
0 & 0 & 0 & d_{15} & 0 & 0 \\
d_{31} & d_{31} & d_{33} & 0 & 0 & 0
\end{array}\right]\left\{\begin{array}{l}
\sigma_{x} \\
\sigma_{y} \\
\sigma_{z} \\
\tau_{y z} \\
\tau_{x z} \\
\tau_{x y}
\end{array}\right\} \\
+\left[\begin{array}{ccc}
\check{n}_{11}^{T} & 0 & 0 \\
0 & \check{n}_{11}^{T} & 0 \\
0 & 0 & \check{n}_{33}^{T}
\end{array}\right]\left\{\begin{array}{l}
E_{x} \\
E_{y} \\
E_{z}
\end{array}\right\}
\end{aligned}
$$

where $\varepsilon_{i}$ represents $i$-th component of normal strain, $\gamma_{i j}$ is $i j$-th component of shear strain, $s_{i j}$ is $i j$-th component of the compliance tensor measured at a constant electric field, $\sigma_{i}$ is $i$-th component of normal stress, $\tau_{i j}$ is $i j$-th component of shear stress, $d_{i j}$ is $i j$-th component of the piezoelectric charge coefficient matrix, $E_{i}$ is $i$-th component of electric field intensity, $D_{i}$ is $i$-th component of electric flux density and $\epsilon_{i j}^{T}$ is ij-th component of permittivity matrix measured at constant mechanical stress.

For a uniaxial stress state Eqs. (1) and (2) reduce to the following form

$$
\begin{aligned}
& \varepsilon_{x}=s_{11}^{E} \sigma_{x}+d_{31} E_{z} \\
& D_{z}=d_{31} \sigma_{x}+\check{n}_{33}^{T} E_{z}
\end{aligned}
$$

By expressing $\sigma_{x}$ from Eq. (3) we obtain

$$
\sigma_{x}=\underbrace{\left(s_{11}^{E}\right)^{-1}}_{Y} \varepsilon_{x}-\underbrace{\left(s_{11}^{E}\right)^{-1} d_{31}}_{e_{31}} E_{z}=Y \varepsilon_{x}-e_{31} E_{z}
$$

where $Y$ is the elastic modulus and $e_{31}=Y \cdot d_{31}$ is the piezoelectric modulus. Inserting the result of Eq. (5) into (4) yields

$$
D_{z}=e_{31} \varepsilon_{x}+\underbrace{\left(\check{n}_{33}^{T}-d_{31} e_{31}\right)}_{\check{r}_{33}^{S}} E_{z}=e_{31} \varepsilon_{x}+\check{n}_{33}^{S} E_{z}
$$

By spatially integrating $D_{z}$ in Eq. (6) over the area of electrodes of $L \times B$ and by assuming that electric field intensity can be approximated with voltage $U$ and electrode distance $h$ as $E_{z}=-U / h$, we obtain the net electric charge $Q$ defined as

$$
Q=\iint_{A_{E}} D_{z} \mathrm{~d} A=\iint_{A_{E}}\left(e_{31} \varepsilon_{X}-\check{\mathrm{r}}_{33}^{s} \frac{U}{h}\right) \mathrm{d} A=e_{31} \iint_{A_{E}} \varepsilon_{X} \mathrm{~d} A-\check{\mathrm{n}}_{33}^{s} \frac{L B}{h} U
$$

where $C_{p}$ is the capacitance of used piezoelectric element. Since the dimensions of used piezoelectric element are small compared to the dimensions of a rail, therefore it is acceptable to assume that strain gradient, i.e. $\partial e_{x} / \partial x$, is negligible and thus Eq. (7) attains the form

$Q=e_{31} \varepsilon_{x} L B-C_{p} U$ 
Next step lies in derivation of Eq. (8) with respect to time

$\frac{\mathrm{d} Q}{\mathrm{~d} t}=e_{31} L B \dot{\varepsilon}_{x}-C_{p} \dot{U}$

Substituting $\mathrm{d} Q / \mathrm{d} t=U / R_{l}$ into Eq. (9) gives the sought relation between the strain rate and voltage generated by piezoelectric element

$\dot{\varepsilon}_{x}=\frac{1}{e_{31} L B}\left(\frac{U}{R_{l}}+C_{p} \dot{U}\right)$

Time-dependent values of strain can be evaluated from (10) as

$\varepsilon_{x}(t)=\frac{1}{e_{31} L B}\left(\frac{1}{R_{l}} \int_{0}^{t} U \mathrm{~d} t+C_{p} U(t)\right)$

\subsection{Laboratory test PVDF and MCF piezoelectric patches on vibrating cantilever beam}

To validate the output of Eq. (10) an experiment with vibrating cantilever beam was carried out. For the testing purposes, following equipment were used: piezoelectric polymer foil type FC 30 from PVDF material manufactured by Piezotech ${ }^{\circledR}$, macrofiber composite (MFC) type M-2807-P2 piezoelectric element manufactured by Smart Material Corp., type 1-LY41-0.6/120 strain gauge made by HBM and a steel cantilever beam with rectangular cross-section of dimensions $465 \times 40 \times 6 \mathrm{~mm}$. Both PVDF and MFC piezoelectric patches, whose electromechanical properties are written in Tab. 1 were glued close to each other to the top surface of the beam near its clamped end, whereas the strain gauge was glued to the bottom surface of the beam at the same distance from clamping (see Fig. 7). The cantilever beam was excited with external impacts on its free end.

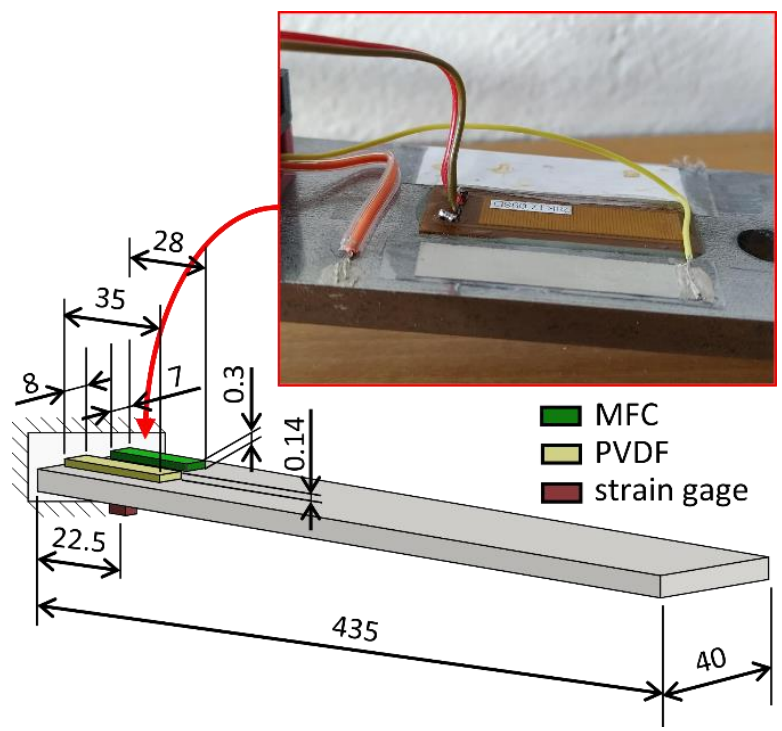

Figure 7. Tested cantilever beam with piezoelectric patches and strain gauge.
The measured signals from piezoelectric elements and used strain gauge are shown in Fig. $8-10$. The voltage output from PVDF piezoelectric element had to be filtered with a lowpass IIR filter due to an excessive amount of high-frequency noise.

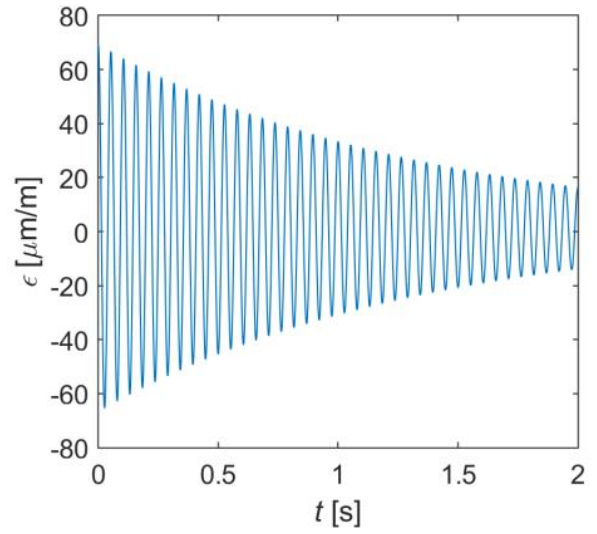

Figure 8. Signal measured by used strain gauge.

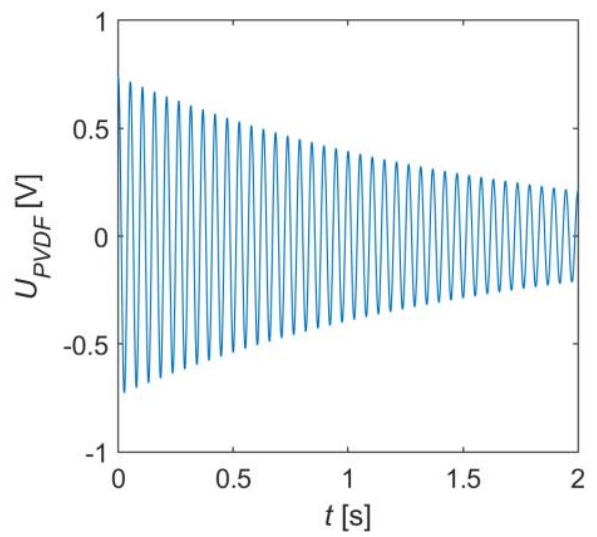

Figure 9. Voltage output measured by PVDF piezoelectric element.

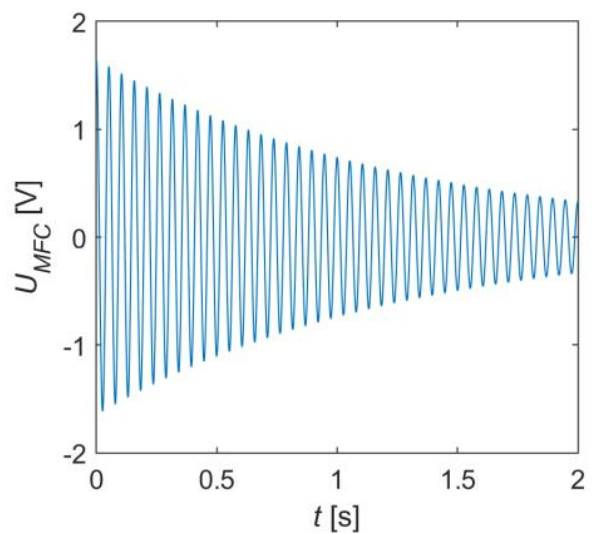

Figure 10. Voltage output measured by MFC piezoelectric element.

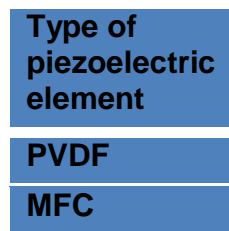

\begin{tabular}{|l|l|l|}
\hline $\begin{array}{l}Y \\
{[\mathrm{GPa}]}\end{array}$ & $\begin{array}{l}\boldsymbol{d}_{31} \\
{[\mathrm{pC} / \mathrm{N}]}\end{array}$ & $\begin{array}{l}\boldsymbol{C}_{p} \\
{[\mathrm{nF}]}\end{array}$ \\
\hline 2 & -20 & 0.21 \\
\hline 30.336 & -170 & 48 \\
\hline
\end{tabular}

\begin{tabular}{|l|l|l|}
\hline $\begin{array}{l}\text { L } \\
{[\mathrm{mm}]}\end{array}$ & $\begin{array}{l}\text { B } \\
{[\mathrm{mm}]}\end{array}$ & $\begin{array}{l}\boldsymbol{h} \\
{[\mathrm{mm}]}\end{array}$ \\
\hline 35 & 8 & 0.14 \\
\hline 28 & 7 & 0.3 \\
\hline
\end{tabular}


Then, since Eq. (10) works with time derivates of strain and voltage, the strain gauge signal was fitted with function $\varepsilon=68 \cdot 10^{-6} \cdot \cos (2 \pi \cdot 19 \cdot t) \cdot e^{-0.75 \cdot t}$, the PVDF voltage output with function $U_{P V D F}=0.74 \cdot \cos (2 \pi \cdot 19 \cdot t) \cdot e^{-0.75 \cdot t}$ and MFC voltage output with function $U_{M F C}=1.65 \cdot \cos (2 \pi \cdot 19 \cdot t) \cdot e^{-0.75 \cdot t}$. Coefficients of these fitting functions were found using Matlab Curve Fitting Toolbox.

For the validation of the model with the experimental test, the output from PVDF piezoelectric element was chosen and results are presented. The result of piezoelectric model Eq. (10) for PVDF voltage signal was compared with measured strain gauge signal, both signals are shown in Fig. 11. The blue curve represents the calculated strain rate from PVDF voltage output using model Eq. (10) and the orange curve represents numerical derivation of strain gauge signal with respect to time.

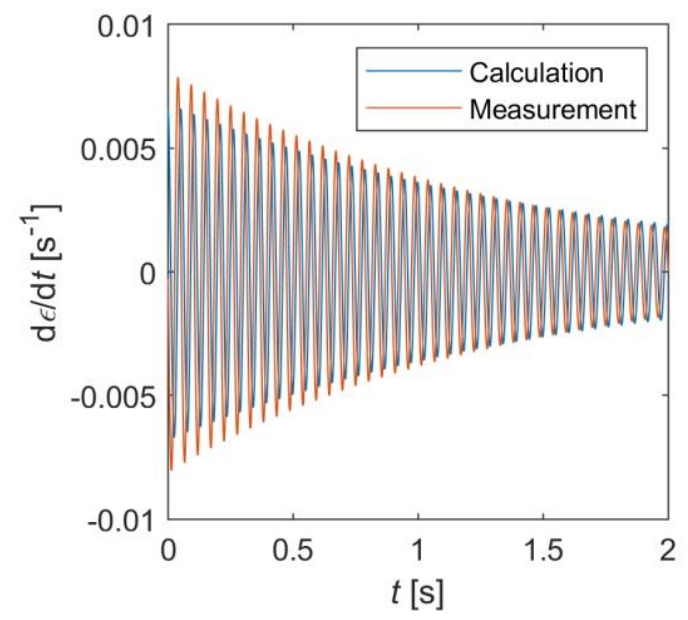

Figure 11. Comparison of calculated and measured strain rate.

There is a very good match in both curves, slight discrepancies near the beginning are caused by lowpass filtering of PVDF voltage signal. Therefore, piezoelectric elements can be successfully used as strain rate sensors and it could be used in sensing applications. However, better voltage outputs will be achieved using MFC piezoelectric elements due to their much higher voltage output compared with PVDF at the same strain levels which in practice means better sensitivity.

\section{EXPERIMENTAL MEASUREMENT WITH A MOVING TRAIN ON A RAIL TRACK}

The piezoelectric MFC patches of dimensions $L \times B \times h(28 \times 7 \times 0.3)$ is attached to the rail between two sleepers as depicted in Fig. 12 and in detail of real application in Fig. 13. This position of the piezoelectric element between sleepers is the most effective for sensing purposes, since maximal strain values occur between the sleepers and, at the same time, it allows for estimation of maximal rail deflection. A pair of piezoelectric MFC was used, see the detail of a rail foot in Fig. 13, due to sensing and energy harvesting purposes [Hadas 2014].

Only the analysis of sensing signal [Hadas 2019] is presented in this paper. The main aim of this chapter is to analyze the voltage signal and estimate the rail deformation, which could be compared with results shown in Fig. 6. This experiment was done in parallel with acceleration measurement for the same passing regional train.

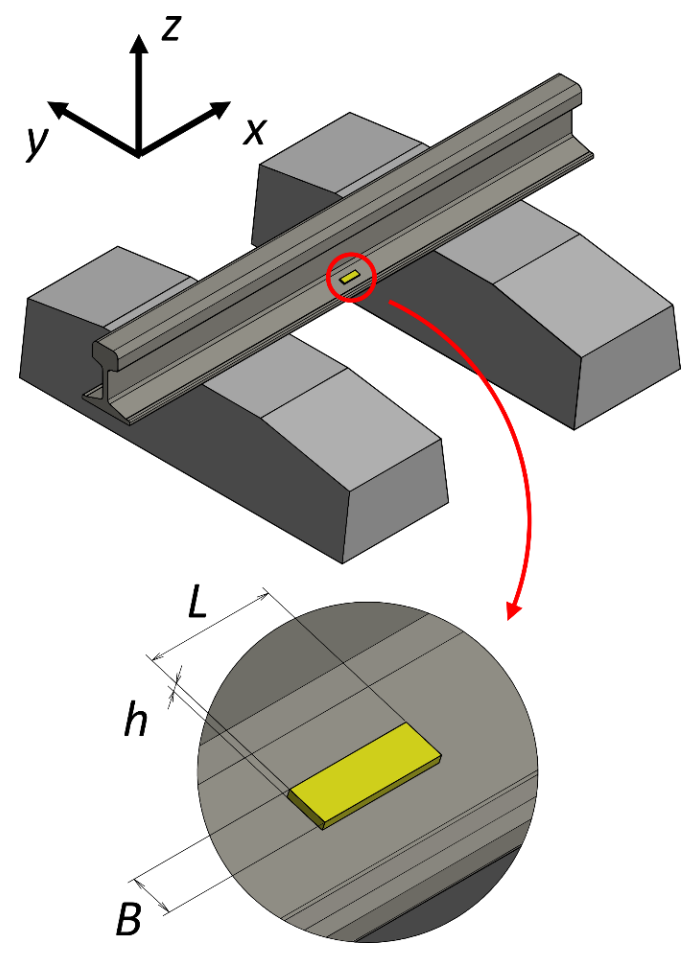

Figure 12. Scheme of a piezoelectric element connected to a rail.

\subsection{Voltage response of piezoelectric patch under passing train}

The location of individual M2807-P2 sensors is displayed in Fig. 13. Used macrofibre composite (MFC) piezoelectric patches made by Smart Material Corp. for data acquisition of active voltage signal the NI DAQ equipment is used. An input channel impedance of this DAQ card is $R_{l}=305 \mathrm{k} \Omega$. The measured signal is shown in Fig. 14. The voltage response corresponds with a single car regional train passing in speed around $27 \mathrm{~km} / \mathrm{h}$.

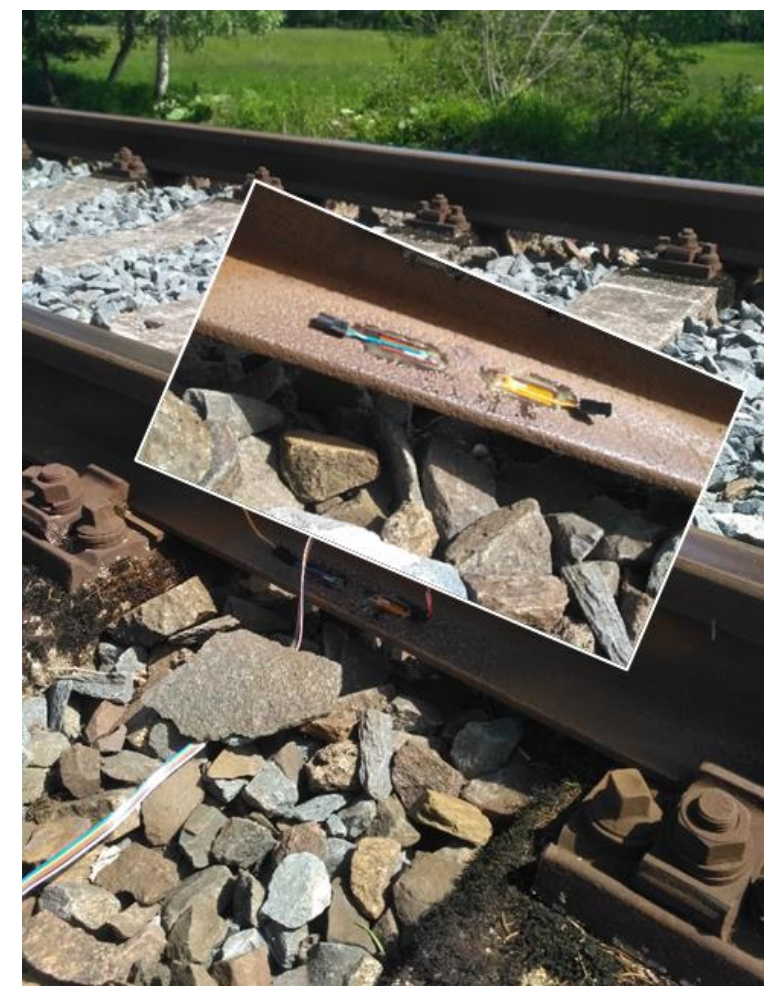

Figure 13. Piezoelectric MFC patches M2807-P2 on rail; detail of a rail foot. 


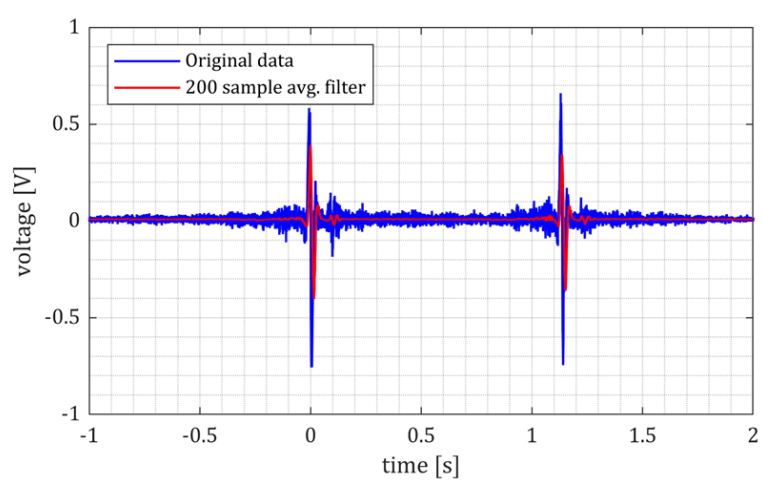

Figure 14. Measured voltage response of MFC patch on rail.

\subsection{Evaluation of rail deformation under moving train}

The voltage output of the MFC piezoelectric patch in Fig. 14. can be used in Eq. (11) to obtain the value of strain on the rail between both sleepers. To estimate the displacement, the rail segment between two sleepers can be thought of as a simply supported beam with $L=690 \mathrm{~mm}$ loaded in the middle with a single force $F$, as shown in Fig. 15. Supports of the beam are characterized by their stiffness $k=60 \mathrm{MN} / \mathrm{m}$; this value is taken from [Gao 2016].
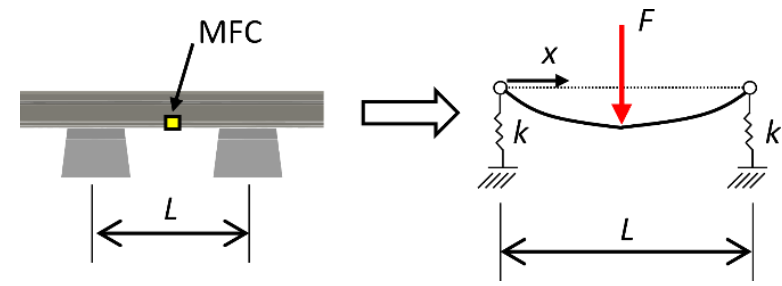

Figure 15. The rail model used for deformation estimation.

Utilizing symmetry of given problem, the transverse displacement is governed by the following differential equation

$Y I \cdot \frac{\mathrm{d}^{4} w}{\mathrm{~d} x^{4}}=0$

with boundary conditions

$$
\begin{aligned}
w(0) & =\frac{F}{2 k} & \frac{\mathrm{d} w}{\mathrm{~d} x}(0.5 \cdot L) & =0 \\
\frac{\mathrm{d}^{2} w}{\mathrm{~d} x^{2}}(0) & =0 & \frac{\mathrm{d}^{3} w}{\mathrm{~d} x^{3}}(0.5 \cdot L) & =-\frac{1}{2} \frac{F}{Y l}
\end{aligned}
$$

where $Y I$ is the rail's bending stiffness estimated as $1.6737 \cdot 10^{8} \mathrm{Nm}^{2}$ using CAD software Autodesk Inventor. Force $F$ can be expressed by the internal bending moment $M$ acting at $x=L / 2$ as

$M=\frac{F}{2} \cdot \frac{L}{2}$

This internal bending moment can be expressed by the calculated strain $e_{x}$ from Eq. (11) as

$M=-Y I \cdot \frac{\mathrm{d}^{2} w}{\mathrm{~d} x^{2}}=Y I \cdot \frac{\varepsilon_{X}}{z_{n}}$

where $z_{n}$ is the distance of piezoelectric element from the neutral axis of the rail cross-section. The value of $z_{n}$ was estimated as $55.5 \mathrm{~mm}$ using commercial CAD software Autodesk Inventor. Combining Eqs. (14) and (15) yields

$$
\frac{F}{2}=2 \cdot Y I \cdot \frac{\varepsilon_{x}}{L \cdot Z_{n}}
$$

By solving equation system (12) and (13), the displacement between both sleepers can be expressed as

$w\left(\frac{L}{2}\right)=2 \cdot \frac{Y I}{k} \frac{\varepsilon_{x}}{L \cdot z_{n}}+\frac{L^{2}}{12} \frac{\varepsilon_{x}}{z_{n}}$

Using Eq. (11) on filtered data presented in section 5 leads to strain values shown in Fig. 16. Using the calculated strain in Eq. (17) yields rail displacement shown in Fig. 17 with a maximal value of $1.16 \mathrm{~mm}$.

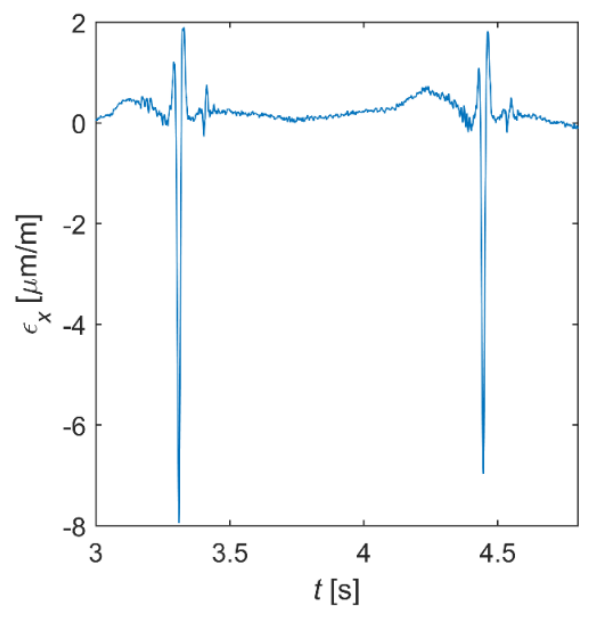

Figure 16. Calculated strain from MFC voltage output.

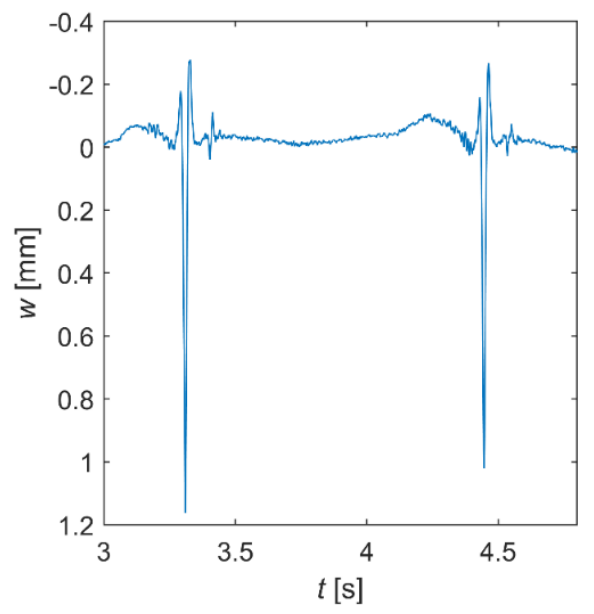

(b)

Figure 17. Calculated rail deformation for experimental measurement.

Both results in Fig. 6 and Fig. 17 provide information about position of rail under moving load of passing regional train. The application of piezoelectric patch on rail provide more accurate position then accelerometers signal due to double integration error.

\section{CONCLUSIONS}

Main aim of this paper is to present advanced piezoelectric sensing system for modern railway monitoring purposes. Both MFC and PVDF patches were tested in the lab environment and compared with strain gauge signal. The presented piezoelectric MFC patch can detect strain of the rail under passing train and used presented theory to estimation of rail deformation. It provides better estimation than double integration of acceleration signal. 
The piezoelectric polymer PVDF could be also integrated on current rails and the installation will be very easy and cheap. PVDF layer provides lower voltage signal however it is suitable for presented application too. This piezoelectric monitoring provides useful tool for affordable sensing system which be integrated in many Industry 4.0 application.

Future development will be aimed to integration of data acquisition and signal processing to autonomous unit which could operate in wireless mode. This modern loT (internet of things) solution could be easy integrated on current rails for detail monitoring of track conditions.

\section{ACKNOWLEDGMENTS}

This presented research was supported by the Brno University of Technology project no. FAST/FSI-J-20-6265.

In case of piezoelectric issues the presented development was supported by the Czech Science Foundation project GA19174575 „Manufacturing and analysis of flexible piezoelectric layers for smart engineering".

\section{REFERENCES}

[@Smart-Materials Corp. 2013] @Smart-Materials Corp. Macro Fiber Composite - datasheet. [online]. 2013 Available from $<$ https://www.smart-

material.com/media/Datasheets/MFC_V2.4-datasheetweb.pdf>

[Abir 2016] Abir, J., et al. Optimized estimator for real-time dynamic displacement measurement using accelerometers. Mechatronics, October 2016, Vol. 39, pp 1-11. ISSN 0957-4158.

[Akyildiz 2002] Akyildiz, I. F., et al. Wireless sensor networks: a survey. Computer Networks, October 2002, Vol. 38, No. 4, pp 393-422. ISSN 1389-1286.

[Andrejašic 2008] Andrejašic, M. MEMS ACCELEROMETERS. University of Ljubljana, Faculty for mathematics and physics, Department of physics.

[Barke and Chiu 2006] Barke, D. W. and Chiu, W. K. A Review of the Effects of Out-Of-Round Wheels on Track and Vehicle Components: Proceedings of the Institution of Mechanical Engineers, Part F: Journal of Rail and Rapid Transit, October 2006.

[Brandt and Brincker 2014] Brandt, A. and Brincker, R. Integrating time signals in frequency domain - Comparison with time domain integration. Measurement, October 2014, Vol. 58, pp 511-519. ISSN 0263-2241.

[Chen 2018] Chen, H.-P. Structural Health Monitoring of Large Civil Engineering Structures. (1st ed.). John Wiley \& Sons, Ltd, October 2018. ISBN 978-1-119-16643-6.

[Cochran 2012] Cochran, S. Piezoelectricity and basic configurations for piezoelectric ultrasonic transducers. In: K. Nakamura, ed. Ultrasonic Transducers: Materials and Design for Sensors, Actuators and Medical Applications. Woodhead Publishing, 2012. ISBN 9781845699895.

[Gao 2016] Gao, M. Y., et al. A rail-borne piezoelectric transducer for energy harvesting of railway vibration. Journal of Vibroengineering, 2016, Vol. 18, No. 7, pp 4647-4663. ISSN 1392-8716.

[Hadas 2014] Hadas, Z., et al. Energy analysis of energy harvesting from machine tool vibrations. MM Science Journal,

\section{4, No. March 2014 ISSN 18050476.}

[Hadas 2019] Hadas, Z., et al. Piezoceramic patches for energy harvesting and sensing purposes. European Physical Journal: Special Topics, 2019, Vol. 228, No. 7, pp 1589-1604.

[Hodge 2015] Hodge, V. J., et al. Wireless Sensor Networks for Condition Monitoring in the Railway Industry: A Survey. IEEE Transactions on Intelligent Transportation Systems, 2015, Vol. 16, No. 3, pp 1088-1106. ISSN 1558-0016.

[Kholkin 2008] Kholkin, A. L., et al. Piezoelectricity and crystal symmetry. In: A. Safari and E. K. Akdoğan, eds. Piezoelectric and Acoustic Materials for Transducer Applications. Boston, MA: Springer, 2008. ISBN 9780387765389.

[Koetse and Rietveld 2009] Koetse, M. J. and Rietveld, P. The impact of climate change and weather on transport: An overview of empirical findings. Transportation Research Part D: Transport and Environment, October 2009, Vol. 14, No. 3, pp 205-221. ISSN 1361-9209.

[Krebs 2015] Krebs, P. High Performances MeMs Accelerometers Are Used in Railway Applications. Railway Technology International;Advanced Electronics, 2015.

[I. Lee 2005] Lee, I., et al. Development and analysis of the vertical capacitive accelerometer. Sensors and Actuators A: Physical, October 2005, Vol. 119, No. 1, pp 8-18. ISSN 09244247.

[J. Lee 2015] Lee, J., et al. A Cyber-Physical Systems architecture for Industry 4.0-based manufacturing systems. Manufacturing Letters, 2015, Vol. 3, pp 18-23. ISSN 22138463.

[Li 2017] Li, C., et al. An overview: modern techniques for railway vehicle on-board health monitoring systems. Vehicle System Dynamics, October 2017, Vol. 55, No. 7, pp 1045-1070. ISSN 0042-3114.

[Lucero 2019] Lucero, P., et al. Accelerometer Placement Comparison for Crack Detection in Railway Axles Using Vibration Signals and Machine Learning. In: 2019 Prognostics and System Health Management Conference (PHM-Paris), pp 291-296.

[Meitzler 1988] Meitzler, A. 176-1987 IEEE standard on piezoelectricity. IEEE, 1988. ISBN 9781504401807.

[Milne 2016] Milne, D., et al. Proving MEMS Technologies for Smarter Railway Infrastructure. Procedia Engineering, October 2016, Vol. 143, pp 1077-1084. ISSN 1877-7058.

[Milne 2018] Milne, D., et al. Automated processing of railway track deflection signals obtained from velocity and acceleration measurements. Proceedings of the Institution of Mechanical Engineers, Part F: Journal of Rail and Rapid Transit, October 2018, Vol. 232, No. 8, pp 2097-2110. ISSN 0954-4097.

[Ngamkhanong 2018] Ngamkhanong, C., et al. State-of-the-Art Review of Railway Track Resilience Monitoring. Infrastructures, October 2018, Vol. 3, No. 1, p 3.

[Oslakovic 2012] Oslakovic, I. S., et al. Climate Change and Infrastructure Performance: Should We Worry About? Procedia - Social and Behavioral Sciences, October 2012, Vol. 48, pp 17751784. ISSN 18770428.

[Park 2005] Park, K.-T., et al. The determination of bridge displacement using measured acceleration. Engineering Structures, October 2005, Vol. 27, No. 3, pp 371-378. ISSN 01410296.

[Pereira 2019] Pereira, L. Trans-European Transport Network 
(TEN-T). Mobility and Transport - European Commission.

[Piezotech ${ }^{\circledR}$ 2020] Piezotech $^{\circledR}$ Piezoelectric Materials | P(VDFTRFE) | Piezotech ${ }^{\circledR}$. [online]. 2020 Available from $<$ https://www.piezotech.eu/en/Products/piezoelectriccopolymers/>.

[Płaczek and Kokot 2019] Płaczek, M. and Kokot, G. Modelling and laboratory tests of the temperature influence on the efficiency of the energy harvesting system based on MFC piezoelectric transducers. Sensors (Switzerland), 2019, Vol. 19, No. 7.

[Qing 2019] Qing, X., et al. Piezoelectric transducer-based structural health monitoring for aircraft applications. Sensors (Switzerland), 2019, Vol. 19, No. 3.

[Salameh 2018] Salameh, J. P., et al. Gearbox condition monitoring in wind turbines: A review. Mechanical Systems and Signal Processing, October 2018, Vol. 111, pp 251-264. ISSN 0888-3270.

[Sappati and Bhadra 2018] Sappati, K. K. and Bhadra, S. Piezoelectric polymer and paper substrates: A review. Sensors (Switzerland), 2018, Vol. 18, No. 11.

[Sirohi and Chopra 2000] Sirohi, J. and Chopra, I. Fundamental Understanding of Piezoelectric Strain Sensors. Journal of Intelligent Material Systems and Structures, April 2000, Vol. 11, No. 4, pp 246-257. ISSN 1045-389X.
[Tang 2018] Tang, X., et al. Energy Harvesting Technologies for Achieving Self-Powered Wireless Sensor Networks in Machine Condition Monitoring: A Review. Sensors, October 2018, Vol. 18, No. 12, p 4113.

[Thong 2004] Thong, Y. K., et al. Numerical double integration of acceleration measurements in noise. Measurement, 2004, Vol. 36, No. 1, pp 73-92. ISSN 02632241.

[Trifunac and Todorovska 2001] Trifunac, M. D. and Todorovska, M. I. A note on the useable dynamic range of accelerographs recording translation. Soil Dynamics and Earthquake Engineering, October 2001, Vol. 21, No. 4, pp 275-286. ISSN 0267-7261.

[Yang 2006] Yang, J., et al. A simple approach to integration of acceleration data for dynamic soil-structure interaction analysis. Soil Dynamics and Earthquake Engineering, October 2006, Vol. 26, No. 8, pp 725-734. ISSN 0267-7261.

[Zhang 2015] Zhang, X., et al. Railway track condition monitoring based on acceleration measurements. In: The 27th Chinese Control and Decision Conference (2015 CCDC), pp 923-928.

[Zhu 2015] Zhu, Y., et al. An Accurate Integral Method for Vibration Signal Based on Feature Information Extraction. Shock and Vibration.

\section{CONTACTS}

Ing. Martina Kratochvilova

Institute of Computer Aided Engineering and Computer Science, Faculty of Civil Engineering, Brno University of Technology Veveri 331/95, Brno, 602 00, Czech Republic

+420 54114 7253, kratochvilova.m@fce.vutbr.cz 